\title{
ON THE BEST CONSTANT FOR THE BESICOVITCH COVERING THEOREM
}

\author{
ZOLTÁN FÜREDI AND PETER A. LOEB
}

(Communicated by James West)

\begin{abstract}
This note shows that in terms of known proofs of the Besicovitch Covering Theorem, the best constant for that theorem is the maximum number of points that can be packed into a closed ball of radius 2 when the distance between pairs of points is at least 1 and one of the points is at the center of the ball. Exponential upper and lower bounds are also established.
\end{abstract}

\section{INTRODUCTION}

Besicovitch's Covering Theorem [4] is more powerful than the familiar result of Vitali because it works for every Borel measure. As the following statement of the theorem indicates, the result itself is independent of measure-theoretic considerations. The usual setting is a finite-dimensional normed vector space $\mathscr{N}=(X,\|\cdot\|)$; here, $B(\mathbf{a}, r)$ denotes a closed ball with center a and radius $r>0$. Fix an arbitrary set $A \subseteq X$ and associate a ball $B(\mathbf{a}, r(\mathbf{a}))$ to each $\mathbf{a} \in A$ so that $\sup _{\mathbf{a} \in A} r(\mathbf{a})<\infty$. The theorem states that there exists a constant, $\beta=\beta(\mathcal{N})$, depending only on the normed space, such that for some $m \leq \beta$, one can find $m$ disjoint subsets $A_{i} \subseteq A$ with the property that for each set $A_{i}$, the associated balls are pairwise disjoint and the union $\cup_{1 \leq i \leq m} \cup_{\mathbf{a} \in A_{i}} B(\mathbf{a}, r(\mathbf{a}))$ still covers $A$.

Besicovitch proved this result for disks in the plane in 1945 [4], and Morse extended it to more general spaces and shapes in 1947 [18]. (For a simple proof of Morse's result, see [6].) The principal use of such covering theorems in analysis is to show that "undesirable sets" are null sets with respect to some $\sigma$-finite Borel measure $\mu$.

Here is an example: Let $S$ be a set contained in the support of $\mu$ with $\mu(S)>0$, and let $\nu$ be a finite Borel measure such that $\nu(S)=0$. To prove the Lebesgue Differentiation Theorem as in [6], one must show that for $\mu$ almost every a $\in S$ there is a $\delta>0$ such that if $0<r<\delta$, then $\mu(B(\mathbf{a}, r)) \geq$ $\nu(B(\mathbf{a}, r))$. Let $A$ be the set of points where this is not true; fix $\varepsilon>0$ and a compact set $K$ in $X \backslash S$ such that $\nu(X \backslash K)<\varepsilon / \beta$. Each a in $A$ is the center

Received by the editors October 28, 1992; presented to the Society at the April 1994 meeting in Brooklyn, NY.

1991 Mathematics Subject Classification. Primary 28A75; Secondary 05B40, 49Q15, 68R 10.

Key words and phrases. Besicovitch Covering Theorem, sphere packings, proximity graphs.

The second author's work was supported by a grant from the National Science Foundation. 
of a ball $B(\mathbf{a}, r) \subset X \backslash K$ such that $\mu(B(\mathbf{a}, r))<\nu(B(\mathbf{a}, r))$. From the $m$ subsets $A_{i}$ of $A$ given by the Besicovitch Covering Theorem, we choose one, $A_{k}$, which maximizes the sum $\sum_{\mathbf{a} \in A_{k}} \mu(B(\mathbf{a}, r(\mathbf{a})))$. Now the outer measure

$$
\begin{aligned}
\mu^{*}(A) & \leq \beta \cdot \sum_{\mathbf{a} \in A_{k}} \mu(B(\mathbf{a}, r(\mathbf{a}))) \\
& \leq \beta \cdot \sum_{\mathbf{a} \in A_{k}} \nu(B(\mathbf{a}, r(\mathbf{a})))<\beta \cdot(\varepsilon / \beta)=\varepsilon .
\end{aligned}
$$

The domination of $\mu^{*}(A)$ by a constant times the total measure of a disjoint set of balls is typical in arguments using covering theorems. Similar calculations are applied in working with maximal functions. The above argument can be modified by selecting a finite subset of $A_{k}$ which works for the constant $2 \beta$. Setting the corresponding balls aside, one can repeat the procedure until all of $A$ except a set of $\mu$-measure 0 has been covered by a countable disjoint set of balls. Another possibility is to use all of the balls in the original subcovering of $A$. Here, one exploits the fact that the sum of the characteristic functions of these balls is bounded by $\beta$.

All known proofs of the Besicovitch Covering Theorem use geometric arguments to establish an upper bound for the cardinality of certain configurations of balls defined in terms of a parameter $\tau>1$. In the next section, we give a definition for this configuration called, as in [15], a $\tau$-satellite configuration of balls. We use $\sigma(\mathscr{N}, \tau)$ to denote the maximum number of balls that can form a $\tau$-satellite configuration, and let $\sigma(\mathscr{N})$ denote $\sigma(\mathscr{N}, 1)$.

Once a geometric bound is established, proofs of Besicovitch's theorem proceed with nongeometric arguments, such as in [15], to show that for $\tau>1$,

$$
\beta(\mathscr{N}) \leq \sigma(\mathcal{N}, \tau) .
$$

For completeness, we will sketch a proof of Equation (1) in Section 5.1. An easy proof of Besicovitch's theorem is formed by the combination of this argument with the proof of Theorem 2.1. The use of ordered satellite configurations as defined in Section 5.2 yields a further simplification.

The principal result of this article, Theorem 2.1, shows that $\sigma(\mathscr{N})$ equals the packing number $\vartheta(\mathscr{N})$ described in the abstract. It also shows that for $\tau$ sufficiently close to $1, \sigma(\mathscr{N}, \tau)=\sigma(\mathscr{N})$. Together with Equation (1), this gives the following result.

Corollary of Theorem 2.1. $\beta(\mathcal{N}) \leq \vartheta(\mathscr{N})$.

Our proof that $\vartheta(\mathscr{N})=\sigma(\mathscr{N})$ is an extension (and simplification) of the first part of results established independently by Reifenberg in [20] and Bateman and Erdös in [3]. They prove that for $E^{2}$, the plane with Euclidean norm,

$$
\vartheta\left(E^{2}\right)=\sigma\left(E^{2}, 1\right) \text { and } \vartheta\left(E^{2}\right)=19 .
$$

We will show that the equality $\vartheta(\mathscr{N})=\sigma(\mathscr{N})$ is valid for every finite dimension and every norm.

Along with our principal result, we have included in this article an exponential lower and upper bound for $\beta$ and $\sigma$. Some of the most important spaces, such as Euclidian space $E^{d}$ and the maximum norm space $\ell_{\infty}^{d}$, are discussed in some detail. 


\section{SATELlite CONFIGURATIONS AND PACKING OF SPHERES}

Fix a constant $\tau \geq 1$. A set of balls $\left\{B\left(c_{i}, r_{i}\right): 0 \leq i \leq n\right\}$ is said to be in $\tau$-satellite configuration with center $B\left(c_{0}, r_{0}\right)$ if the following conditions hold for $i \geq 1$ :

(i) $B\left(\mathbf{c}_{0}, r_{0}\right) \cap B\left(\mathbf{c}_{i}, r_{i}\right) \neq \varnothing$,

(ii) $r_{0} \leq \tau \cdot r_{i}$,

(iii) $\tau \cdot\left\|\mathbf{c}_{i}-\mathbf{c}_{0}\right\| \geq \max \left(r_{i}, r_{0}\right)$, and

(iv) if $1 \leq i<j \leq n$, then either $\left\|\mathbf{c}_{i}-\mathbf{c}_{j}\right\| \geq r_{i} \geq r_{j} / \tau$ or $\left\|\mathbf{c}_{i}-\mathbf{c}_{j}\right\| \geq r_{j} \geq$ $r_{i} / \tau$.

Let $\sigma(\mathscr{N}, \tau)$ denote the least upper bound for the number of balls in any $\tau$-satellite configuration in $\mathscr{N}$, and set $\sigma(\mathcal{N})=\sigma(\mathscr{N}, 1)$. Clearly, $\sigma(\mathcal{N}, \tau)$ is an increasing function of $\tau$. Present proofs of the Besicovitch Covering Theorem in the literature show that for $1<\tau \leq 2, \sigma(\mathscr{N}, \tau)$ is a finite (natural) number which plays the role of an upper bound for the number of families of disjoint balls given by that theorem.

Let $\vartheta=\vartheta(\mathscr{N})$ be the maximum number of points in a set $P$ that can be packed into the closed ball $B(0,2)$ with the distance between pairs of distinct points at least 1 and with one of the points fixed at the origin. Such a set $P$ is called a centered point packing into a ball of radius 2 . Let $P$ be such a packing with cardinality $|P|=\vartheta$. By centering a ball of radius $1 / 2$ about each of the points of $P$, one easily verifies the well-known fact that $\vartheta$ is at most the volume of $B(0,5 / 2)$ divided by the volume of $B(0,1 / 2)$, whence, in terms of the dimension $d$ of $X$,

$$
\vartheta \leq 5^{d}
$$

On the other hand, centering a ball of radius 1 about each point of $P$ forms a 1-satellite configuration, so

$$
\vartheta(\mathscr{N}) \leq \sigma(\mathcal{N}) .
$$

It is easy to show with a simple compactness argument that it is still true for a small $\delta=\delta(\mathscr{N})>0$ that at most $\vartheta$ points can be packed into the closed ball $B(0,2)$ when the distance between pairs of distinct points is at least $1-\delta$ and one of the points is fixed at the origin (e.g., see [15]). We will establish the following result in Section 7.

2.1 Theorem. Fix a set of balls $B\left(c_{i}, r_{i}\right), \quad 0 \leq i \leq n$, satisfying conditions (i)-(iv). If $1 \leq \tau<1+\frac{\delta}{4}$, then $n+1 \leq \vartheta$, i.e., $\sigma(\mathcal{N}, \tau) \leq \vartheta(N)$. Thus, by Equation (4),

$$
\vartheta(\mathscr{N})=\sigma(\mathscr{N})=\sigma(\mathscr{N}, \tau) .
$$

\section{ESTIMATES FOR SPHERE PACKING IN HIGHER DIMENSIONS}

3.1 Proposition. The order of magnitude of $\vartheta\left(E^{d}\right)$ is exponential in $d$ :

$$
\left(\frac{8}{\sqrt{15}}+o(1)\right)^{d} \sim(2.065 \cdots+o(1))^{d} \leq \vartheta\left(E^{d}\right) \leq(2.691 \cdots+o(1))^{d} .
$$

Proof. For these calculations, we let $M(d, \varphi)$ and $N(d, \varphi)$ denote, respectively, the maximum number of points on the surface and inside the ball $B(0,1)$ in Euclidian space $E^{d}$, when on the surface, all of the mutual angular distances 
are greater than or equal to $\varphi$, and inside the ball, the Euclidean distances are at least $2 \sin (\varphi / 2)$. Obviously, $M(d, \varphi) \leq N(d, \varphi)$. Lower bounds for $M(d, \varphi)$ were given by Shannon [21] in 1959:

$$
\left(\frac{1}{\sin \varphi}+o(1)\right)^{d} \leq M(d, \varphi) \text {. }
$$

The best known upper bound for $N(d, \varphi)$ was obtained by Kabatjanskii and Levenštein [13]: For all fixed $\varphi<62^{\circ}$

(7) $\frac{1}{d} \log N(d, \varphi) \leq \frac{1+\sin \varphi}{2 \sin \varphi} \log \frac{1+\sin \varphi}{2 \sin \varphi}-\frac{1-\sin \varphi}{2 \sin \varphi} \log \frac{1-\sin \varphi}{2 \sin \varphi}+o(1)$.

The bounds in Equation (5) follow from Equations (6) and (7) using the value $\sin (\varphi / 2)=1 / 4$.

Consider a centered point packing $P$ into the ball $B(0,2)$ in the Euclidean space $E^{d}$. Define $\varphi_{i}, i=1,2$, by $\sin \left(\varphi_{1} / 2\right)=1 / 4$ and $\sin \left(\varphi_{2} / 2\right)=1 / 3(0<$ $\left.\varphi_{i}<90^{\circ}\right)$. Split $P$ into 3 parts: the center, the points contained in $B(0,1.5) \backslash$ $\{0\}$, and the rest. One can easily see that there are, respectively, at most 1 , $M\left(d, \varphi_{2}\right)$, and $M\left(d, \varphi_{1}\right)$ points in these parts. Here $M\left(d, \varphi_{2}\right) \leq(2.004 \cdots+$ $o(1))^{d}$ which is less than the left-hand side of Equation (5), hence $\vartheta=(1+$ $o(1)) M\left(d, \varphi_{1}\right)$. In the same way, it is easy to see that in general the functions $N$ and $M$ have the same order of magnitude, $N(d, \varphi) \leq M(d, \varphi) O\left(\varphi^{-3}\right)$.

In the case of the Euclidean metric, our main Theorem 2.1 was independently proved by Sullivan in his thesis (also see his forthcoming paper [22]), along with the estimates in (5). Using the Mathematica program he was able to narrow the gap in small dimensions showing that $67 \leq \vartheta\left(E^{3}\right) \leq 87,226 \leq \vartheta\left(E^{4}\right) \leq 331$, $681 \leq \vartheta\left(E^{5}\right) \leq 1159$.

\section{A LOWER BOUND FOR THE Besicovitch CONSTANT}

No proof of the Besicovitch theorem can provide a constant better than the maximum $\kappa=\kappa(\mathcal{N})$ for the number of balls that can form a configuration in $X$ where each ball of the configuration intersects every other ball but does not contain the center of the other ball. We will call such a system an intersecting satellite configuration. For the plane, the value of $\kappa\left(E^{2}\right)$ is at least 8 , as is shown by the following construction due to Malnic and Mohar [29] and independently to E. Scheinerman (see in [12]): The centers of the eight circles are formed by the vertices of a regular pentagon and the three vertices of a small equilateral triangle with the same center such that no three vertices are collinear. It is conjectured that $\kappa\left(E^{2}\right)=8$. Krantz and Parsons [27] showed $\kappa\left(E^{2}\right) \leq 20$; by [3] and [20] one knows that $\kappa\left(E^{2}\right) \leq 19$. In [28] Kézdy and Kubicki showed 11 is an upper bound. In general, let $S$ be a set of points such that all the mutual distances are strictly greater than 1 and at most 2 . Centering a unit ball at each point of $S$ we get an intersecting satellite configuration. If we consider a packing $S$ into the unit ball of $E^{d}$ with $\varphi=60^{\circ}$, then Equations (6) and (7) give

$$
(2 / \sqrt{3}+o(1))^{d} \sim(1.154 \cdots+(1))^{d} \leq|S| \leq(1.3208 \cdots+o(1))^{d} .
$$

The following lower bound for $\kappa\left(E^{d}\right)$ is obtained in the same way as for Equation (5): 
4.1 Proposition. $1.25^{d}<\kappa\left(E^{d}\right) \leq(1.887 \cdots+o(1))^{d}$.

Proof. To see the lower bound, consider the ball $B(0, \sqrt{5} / 2)$ and let $S$ be a set on the surface such that any angular distance between two points is more than $\varphi_{3}$ and less than $\pi-\varphi_{3}$ where $\sin \varphi_{3}=4 / 5$. One can construct such a set $S$ by taking the points $\mathbf{p}_{1}, \ldots, \mathbf{p}_{m}$ one by one from the surface. One can continue this process until the area of the two caps with angular radii $\varphi_{3}$ is less than $1 / m$ of the surface area of the ball. The surface area of these two caps is less than the surface area of a ball of radius $\sin \varphi_{3}(\sqrt{5} / 2)$. The rest of the calculation is left to the reader.

To prove the upper bound, suppose (in somewhat greater generality) that $B\left(\mathbf{c}_{i}, r_{i}\right)$ is a set of balls such that $r_{i}, r_{j} \leq\left\|\mathbf{c}_{i}-\mathbf{c}_{j}\right\| \leq r_{i}+r_{j}$ for all $1 \leq i<$ $j \leq m$. Suppose that $\min r_{i}=r_{1}=1$. Split this satellite configuration into $d^{2}$ subconfigurations employing the radii of the balls in the following way. Let $C_{k}=\left\{\mathbf{c}_{i}:(1+(1 / d))^{(k-1) / 2} \leq r_{i}<(1+(1 / d))^{k / 2}\right\}$ for $k=1,2, \ldots, d^{2}-1$, and let $C_{d^{2}}=\left\{\mathbf{c}_{i}: r_{i} \geq(1+(1 / d))^{d^{2}}\right\}$. Let $R_{i}=\min \left\{r_{i}: \mathbf{c}_{i} \in C_{i}\right\}$. In the case $k<d^{2}$ all distances between the points of $C_{i}$ are at least $R_{i}$ and at most $(2 \sqrt{(d+1) / d}) R_{i}$. By Jung's Theorem (see, e.g., in [25]), any set in $E^{d}$ of diameter $D$ is contained in a ball of radius $D \sqrt{2 d /(d+1)}$. Thus $C_{k}$ is contained in a ball of radius $\sqrt{2} R_{i}$. The upper bound in Equation (7) with $\cos \varphi=3 / 4$ gives $\left|C_{k}\right|<(1.887 \ldots)^{d}$. In the case $k=d^{2}$ the distance $\left\|\mathbf{c}_{1}-\mathbf{c}_{i}\right\|$ $\left(\mathbf{c}_{i} \in C_{k}\right)$ is at least $\exp (d / 2)$. It is easy to see (the details are the same as in the proof of Theorem 2.1 in Section 7), that in this case the angles $\mathbf{c}_{i} \mathbf{c}_{1} \mathbf{c}_{j}$ are at least $60^{\circ}-o(1)$. One can apply the upper bound in Equation (7) again (with $\left.\varphi=60^{\circ}-o(1)\right)$ to get $\left|C_{d^{2}}\right|<(1.3208 \cdots+o(1))^{d}$.

4.2 Conjecture. We think that the above construction is (essentially) best possible; i.e., $\kappa\left(E^{d}\right) \sim M\left(d, \varphi_{3}\right)$. This value of the function $M$ is at most $(1.480 \cdots+$ $o(1))^{d}$, as shown by Equation (7).

For the $d$-dimensional maximum norm space, $\ell_{\infty}^{d}$, we have

$$
\kappa\left(\ell_{\infty}^{d}\right)=2^{d} .
$$

Indeed, any set of cubes, $\mathscr{Q}$, has the Helly property; i.e., if the members of $\mathscr{Q}$ pairwise intersect then all of them have a common point. Suppose this common point is the origin 0 . If two cubes $Q$ and $Q^{\prime}$ from $Q$ have centers in the same orthant, say $0 \leq \mathbf{q}$ and $0 \leq \mathbf{q}^{\prime}$ with $\|\mathbf{q}\| \leq\left\|\mathbf{q}^{\prime}\right\|$, then the center of one of the cubes is contained in the other, $\mathbf{q} \in Q^{\prime}$.

A clever construction of Ajtai [1] shows $\beta\left(\ell_{\infty}^{2}\right) \geq 5$, though $\kappa=4$. The upper bound in Equation (3) is tight for this norm, as one can see by working with the points $\{-2,-1,0,1,2\}^{d}$. That is,

$$
\vartheta\left(\ell_{\infty}^{d}\right)=5^{d} .
$$

In general, we have an immediate lower bound $\Omega\left(d^{2}\right)$ for $\kappa$ using [23]. Recently, J. Bourgain verified the author's conjecture that the true order of magnitude of $\kappa$ (and thus the order of $\beta$ and $\sigma$ ) is exponential in $d$ for any norm. His proof is based on the following result of Milman [17]. For each $c>0$ there exists a $\psi(c)>0$ such that every finite-dimensional normed space $(X,\|\cdot\|)$ of dimension $d$ admits two subspaces $Z \subset Y \subset X$ with the 
properties that $\operatorname{dim}(Z)>\psi(c) d$ and the projection $\pi$ of $Y \cap B$ (where $B$ is the unit ball of $X$ ) into $Z$ is $(1+c)$-equivalent to an ellipsoid in $Z$. That is, there exists an ellipsoid $L \subset \pi(Y \cap B) \subset(1+c) L \subset Z$. Milman's theorem permits the reduction of various problems about general convex bodies to the Euclidean case. A complete exposition of this theory with background may be found in Pisier's recent book [19].

4.3 Theorem (Bourgain). For any number $s<\sqrt{2}$ there exists an $\varepsilon(s)>0$, such that in any normed space of dimension $d$ there is an at least $(1+\varepsilon(s))^{d}$ element point-set $K$ on the unit sphere with the property that the distances between distinct points are at least $s$.

Proof. Fix an arbitrary $c>0$ and a $d$-dimensional space $(X,\|\cdot\|)$. Let $Z \subset Y \subset X$ be the subspaces provided by Milman's theorem. The set $\pi(Y \cap B)$ is $(1+c)$-equivalent to the Euclidean unit ball in $Z$, so there are exponentially many points forming a set $K_{0}$ on the surface of that ball such that the distance between any two points is at least $\sqrt{2}-2 c$. (The last statement follows from Equation (6).) For every $z \in K_{0}$, choose a point $y \in Y$ on the surface of $B$ such that $\pi(y)=z$. These points $y$ form the desired set $K$ in the unit sphere of $X$.

To obtain a lower bound for $\kappa$, we set $s=1.1$. Taking balls with centers in $K$ and radii equal to 1.01 , we get a family all containing the origin. It follows that $\kappa>1.001^{d}$.

In a related problem, $K$. Bezdek [5] conjectures that for any convex body $K \subset R^{d}$, the number of pairwise tangent homothetic copies of $K$ is not more than $2^{d}$. In a theorem of Hadwiger (about the number of translated copies of $K$ tangent to $K$ ) this number is at most $3^{d}$. One can ask the following more general question; other, related problems can be found in [10]:

4.4 Problem. Is it true that for any centrally symmetric body $K$ of dimension $d, d \geq d_{0}$, the number of pairwise intersecting homothetic copies of $K$ which do not contain each other's centers is at most $2^{d}$; i.e., is $\kappa \leq 2^{d}$ ? $\left(\right.$ Since $\kappa\left(E^{2}\right) \geq 8$, we must have $d_{0} \geq 3$.)

\section{ReduCING BesicovitCH'S THEOREM TO A SATELlite PROBLEM}

5.1 A short proof of Equation (1). The proof in [15] that Equation (1) holds (and the proof of the more general result in [6]) starts with a set $A$ and a covering $\mathscr{C}$ formed by balls centered at each point of $A$ with a finite upper bound for the radii. One then finds by a transfinite induction a well-ordered subcovering $\mathscr{C}_{0}=\left\{C_{0}, C_{1}, C_{2}, \ldots\right\}$ of $\mathscr{C}$ by selecting at each stage, $\alpha$, a ball $C_{\alpha}$ with center not yet covered by $\cup_{\gamma<\alpha} C_{\gamma}$ and radius bigger than any competing radius divided by $\tau$. (I.e., $r_{\alpha}>(1 / \tau) \sup \{r(C): C \in \mathscr{C}$, center $(C)$ not yet covered $\}$.) Note that any pair of balls from $\mathscr{C}_{0}$ satisfies condition (iv) (even without equality). The last step is to show that $\mathscr{C}_{0}$ can be decomposed into at most $\sigma(\mathscr{N}, \tau)$ disjoint subfamilies.

The well-ordering is used to extract a maximum disjoint subfamily $\mathscr{C}_{1}$ from $\mathscr{C}_{0}$ by induction as follows. At each stage, the first ball that does not intersect any of the balls already in $\mathscr{C}_{1}$ is removed from $\mathscr{C}_{0}$ and added to $\mathscr{C}_{1}$; the process stops when every ball that remains in $\mathscr{C}_{0}$ intersects some ball in $\mathscr{C}_{1}$. Once $\mathscr{C}_{1}$ 
is found, the process is repeated to extract a maximum disjoint subfamily $\mathscr{C}_{2}$ from $\mathscr{C}_{0} \backslash \mathscr{C}_{1}$ (unless only the empty set remains). Once $\mathscr{C}_{m}$ has been found, if a ball $B_{0}$ remains in $\mathscr{C}_{0}$ and for $1 \leq i \leq m, B_{i}$ is the first ball in $\mathscr{C}_{i}$ to intersect $B_{0}$, then it is easy to see that the balls $\left\{B_{0}, B_{1}, \ldots, B_{m}\right\}$ are in $\tau$-satellite configuration with center $B_{0}$. It follows that $m+1 \leq \sigma(\mathscr{N}, \tau)$.

Using additional assumptions, it is shown in [16] that a subcovering can be extracted from $\mathscr{C}$ with the property that no center is in the interior of any other ball of the subcovering and each point $\mathbf{a} \in A$ is contained in a ball with radius at least as great as the radius of the ball originally centered at $\mathbf{a}$. The setting in [16] is a locally compact metric space. For related results, see [27].

5.2 Ordered satellite configurations. Take the balls $B_{0}, B_{1}, \ldots, B_{m}$ obtained in Section 5.1, and reorder them using the reverse of the well-ordering of $\mathscr{C}_{0}$. Denote the new sequence by $\left\{B^{0}, B^{1}, \ldots, B^{m}\right\}$. It is easy to see that $B^{0}=B_{0}$, and for $0 \leq i<j \leq m$

$$
\left\|\mathbf{c}^{i}-\mathbf{c}^{j}\right\|>r^{j}>r^{i} / \tau \text {. }
$$

We call a system of balls $B^{0}, B^{1}, \ldots, B^{m}$ satisfying Equation (10) with $B^{0} \cap$ $B^{i} \neq \varnothing$ an ordered satellite configuration (with constant $\tau$ ). Apparently, this condition is stronger than the condition of being a satellite configuration, so the maximum size $\sigma_{\text {ord }}(\mathcal{N}, \tau) \leq \sigma(N, \tau)$. However, for sufficiently small $\tau>1$, equality still holds here. Indeed as the following example shows, both quantities are equal to $\vartheta(\mathscr{N})$. Let $P$ be a maximal centered point packing into the ball $B(0,2)$, with $|P|=\vartheta$. Fix a ball of radius $\tau^{1 / 3}$ about 0 and a ball of radius $1 / \tau^{1 / 3}$ about each of the other points of $P$. This is an ordered satellite configuration of size $\vartheta$.

The case $\tau=1$ is somewhat different. For example, by a theorem of Gritzmann [11], the packing resulting in $\beta\left(\ell_{\infty}^{d}\right)=5^{d}$ (see Equation (9)) is unique. However, with these centers one cannot obtain an ordered satellite configuration with $\tau=1$.

5.3 Chromatic number of line graphs. The second part of the above proof of Equation (1) can be summarized in the following well-known coloring lemma (applied to the family $\mathscr{C}_{0}$ ). For an arbitrary family of sets, $\mathscr{S}$, define the graph $L(\mathscr{S})$, the so-called line graph or intersection graph of $\mathscr{S}$, with vertex set $\mathscr{S}$ by joining two members $S_{1}$ and $S_{2}$ if they are not disjoint, i.e., $S_{1} \cap S_{2} \neq$ $\varnothing$. A coloring of the line graph corresponds to a decomposition of $\mathscr{S}$ into subfamilies consisting of mutually disjoint sets. The following lemma (see, e.g., [7]) gives an upper bound for the chromatic number of $L(\mathscr{S})$.

5.4 Lemma. Suppose that for any finite subsystem $\mathscr{S}_{0}$ of $\mathscr{S}$ there is an ordering $\mathscr{S}_{0}=\left\{S_{1}, S_{2}, \ldots, S_{t}\right\}$ such that $S_{i}$ intersects at most $\chi$ of the sets preceding it; i.e., $\left|\left\{S_{j} \cap S_{i} \neq \varnothing: j<i\right\}\right| \leq \chi$. Then for the chromatic number of $L(\mathscr{S})$ we have

$$
\operatorname{chr}(L(\mathscr{S})) \leq \chi+1
$$

Proof. This lemma is a composition of two facts. First, the deBruijn-Erdös lemma, that $\operatorname{chr}(\mathscr{G}) \leq k$ (where $k$ is a positive integer) if $\operatorname{chr}\left(\mathscr{G}^{\prime}\right) \leq k$ for every finite subgraph of $\mathscr{G}$. Second, the chromatic number of $\mathscr{G}^{\prime}$ is at most $\chi+1$ if every subgraph has a vertex of degree at most $\chi$. (In our case, $S_{t}$ is connected to at most $\chi$ sets.) 


\section{THE SPHERE OF INFLUENCE GRAPH}

The results given by Equation (2) were rediscovered several times, frequently in a weaker form. Here we collect some of these related results. Our bounds in higher dimensions can be generalized for these problems.

Let $S$ be a finite set of at least two points in the normed space $\mathcal{N}$. For each point $\mathbf{c} \in S$ let $r(\mathbf{c})$ be the closest distance to any other point in the set, and let $B(\mathbf{c})$ and $A(\mathbf{c})$ be the closed and open balls of radius $r(\mathbf{c})$ centered at $\mathbf{c}$, respectively.

6.1 Definition. The sphere of influence graph of $S$, written as $S I G(S)$, is the intersection graph $L(\{A(\mathbf{c}): \mathbf{c} \in S\})$, i.e., its vertex set is $S$ with $\mathbf{x}$ and $\mathbf{y}$ in $S$ adjacent if and only if their open balls have nonempty intersection, $r(\mathbf{x})+r(\mathbf{y})>\|\mathbf{x}-\mathbf{y}\|$. The closed sphere of influence graph of $S$, written as $C S I G(S)$, is the graph with vertex set $S$ with $\mathbf{x}$ and $\mathbf{y}$ in $S$ adjacent if and only if their closed balls have nonempty intersection.

The definition of $S I G$ 's is due to Toussaint [24]; these graphs have been widely investigated recently. For the Euclidean plane, Equation (2) implies that each $S I G$ on $n$ vertices has at most $18 n$ edges. In [2], the somewhat weaker upper bound $29 n$ was proved together with an algorithmic description of the running time $O(n \log n)$. Independently, in Edelsbrunner, Rote, and Welzl's article [8], 30n was proved in the following more general form. Let $r^{m}(\mathbf{c})$ denote the distance from c to its $m$-nearest neighbors; that is, the closed ball $B\left(\mathbf{c}, r^{m}(\mathbf{c})\right)$ contains at least $m$ more points from $S$, while its interior contains less than $m$. The graph $C S I G^{m}(S)$ is defined as the intersection graph of these balls. In [8] it was proved that this graph always contains a vertex of degree at most $31 m-1$, which, using Equation (2), can be improved to $19 m-1$. Hence, the number of edges is at most $(19 m-1) n$, and its chromatic number is at most $19 \mathrm{~m}$. For higher dimensions, see Guibas, Pach, and Sharir [26]. In general, it is conjectured that for the Euclidean plane a $S I G$ cannot have more than $9 n$ edges.

6.2 Proposition. All of the above results follow from Theorem 2.1, since the ball having the smallest radius and its neighbors form a satellite configuration.

Still another problem has been investigated by Fejes Tóth and Heppes [9]. They prove, that in any planar packing of unit disks no circle can have more than 18 neighbors and second neighbors. They conjecture that with one step more (i.e., counting first, second, and third neighbors), the answer is 36 . For more problems see, e.g., the papers and books of L. Fejes Tóth.

\section{Proof of Theorem}

Proof of Theorem 2.1. We may assume that $\mathbf{c}_{0}=0$ and $r_{0}=1$. Given $0 \leq$ $i \leq n$, if $\left\|\mathbf{c}_{i}\right\| \leq 2$, we set $\mathbf{b}_{i}=\mathbf{c}_{i}$, and if $\left\|\mathbf{c}_{i}\right\|>2$, we replace $\mathbf{c}_{i}$ with the point $\mathbf{b}_{i}=\left(2 /\left\|\mathbf{c}_{i}\right\|\right) \mathbf{c}_{i}$. We will show that $\left\|\mathbf{b}_{i}-\mathbf{b}_{j}\right\|>1-\delta$ for all $i$, $j \leq n$ with $i \neq j$. Consider first the case that $\left\|\mathbf{c}_{i}\right\| \leq 2$ and $\left\|\mathbf{c}_{j}\right\| \leq 2$. By conditions (ii), (iii), and (iv), $\left\|\mathbf{b}_{i}-\mathbf{b}_{j}\right\| \geq 1 / \tau$, which is what is desired, since $0 \leq 1-1 / \tau \leq \tau-1<\delta$. Now consider the case that $\left\|\mathbf{c}_{i}\right\| \leq 2$ and $\left\|\mathbf{c}_{j}\right\|>2$. Since $B\left(\mathbf{c}_{j}, r_{j}\right) \cap B(0,1) \neq \varnothing, B\left(\mathbf{b}_{j}, 1\right) \subseteq B\left(\mathbf{c}_{j}, r_{j}\right)$. If $\left\|\mathbf{c}_{i}-\mathbf{c}_{j}\right\| \geq r_{j}$, then $\left\|\mathbf{b}_{i}-\mathbf{b}_{j}\right\| \geq 1$. On the other hand, if $\tau>1$ and $\left\|\mathbf{c}_{i}-\mathbf{c}_{j}\right\|<r_{j}$, then $\left\|\mathbf{c}_{i}-\mathbf{c}_{j}\right\| \geq$ 
$r_{i} \geq r_{j} / \tau$. By condition (iii), $2 \tau \geq\left\|\mathbf{c}_{i}\right\| \tau \geq r_{i}$, so $2 \tau^{2} \geq r_{j}$. Since $\tau<5 / 4$,

$$
r_{j}-r_{j} / \tau \leq 2 \tau^{2}(1-1 / \tau) \leq 2 \tau^{2}(\tau-1)<\delta,
$$

and so

$$
\left\|\mathbf{b}_{i}-\mathbf{b}_{j}\right\|=\left\|\mathbf{c}_{i}-\mathbf{b}_{j}\right\| \geq\left\|\mathbf{c}_{i}-\mathbf{c}_{j}\right\|-\left\|\mathbf{c}_{j}-\mathbf{b}_{j}\right\|>r_{j}-\delta-\left(r_{j}-1\right)=1-\delta .
$$

Finally, given $i$ and $j$ with $i \neq j$ and $\left\|\mathbf{c}_{i}\right\| \geq\left\|\mathbf{c}_{j}\right\|>2$, we set $s=\left\|\mathbf{c}_{j}\right\|$ and we set $\mathbf{x}=\left(s /\left\|\mathbf{c}_{i}\right\|\right) \mathbf{c}_{i}$. Since

$$
\left\|\mathbf{c}_{i}-\mathbf{c}_{j}\right\| \leq\left\|\mathbf{c}_{i}-\mathbf{x}\right\|+\left\|\mathbf{x}-\mathbf{c}_{j}\right\|=\left\|\mathbf{c}_{i}\right\|-\left\|\mathbf{c}_{j}\right\|+\left\|\mathbf{x}-\mathbf{c}_{j}\right\|,
$$

we have the "Bow and Arrow Inequality"

$$
\left\|\mathbf{x}-\mathbf{c}_{j}\right\| \geq\left(\left\|\mathbf{c}_{j}\right\|+\left\|\mathbf{c}_{i}-\mathbf{c}_{j}\right\|\right)-\left\|\mathbf{c}_{i}\right\| .
$$

By condition (i), $\left\|\mathbf{c}_{i}\right\| \leq r_{i}+1$, so

$$
\left\|\mathbf{x}-\mathbf{c}_{j}\right\| \geq s-1+\left\|\mathbf{c}_{i}-\mathbf{c}_{j}\right\|-r_{i} .
$$

If $\tau>1$ and $\left\|\mathbf{c}_{i}-\mathbf{c}_{j}\right\|-r_{i}<0$, then by conditions (iv) and (iii),

$$
r_{i}-\left\|\mathbf{c}_{i}-\mathbf{c}_{j}\right\| \leq r_{i}-r_{j} \leq r_{j}(\tau-1) \leq \tau s(\tau-1) .
$$

In any case, since $s>2$ and $2 \tau(\tau-1)<\delta$,

$$
\left\|\mathbf{b}_{i}-\mathbf{b}_{j}\right\|=\left\|\frac{2}{s} \mathbf{x}-\frac{2}{s} \mathbf{c}_{j}\right\| \geq 2-\frac{2}{s}-2 \tau(\tau-1)>1-\delta .
$$

\section{Conclusion}

We have shown that in every finite-dimensional normed space,

$$
1.001^{d} \leq \kappa \leq \beta \leq \sigma=\vartheta \leq 5^{d} .
$$

Here, $\kappa$ is the maximum number of balls in $\mathscr{N}$ pairwise meeting each other but not containing each other's center, $\beta$ stands for the best constant in Besicovitch's theorem, $\sigma$ is the maximum number of balls in a satellite configuration, and $\vartheta$ is a packing number for a ball of radius 2 . Each of these numbers grows exponentially with the dimension $d$, and for certain spaces much better estimates can be given. Exact solutions are usually difficult to obtain, however, even in two dimensions.

\section{ADDED IN PROOF}

The authors are indebted to T. S. Michael for helpful comments. He and T. Quint have recently proved that a CSIG of $n$ vertices in the $d$-dimensional space $\mathscr{N}$ has at most $\left(5^{d}-(3 / 2)\right) n$ edges [30].

\section{REFERENCES}

1. M. Ajtai, The solution of a problem of T. Rado, Bull. Acad. Polon. Sci. Ser. Math. Astr. Phys. 21 (1972), 61-63.

2. D. Avis and J. Horton, Remarks on the sphere of influence graph, Proc. Conf. on Discrete Geometry and Convexity (J. E. Goodman et al., eds.), New York, 1982; Ann. New York Acad. Sci., vol. 440, New York Acad. Sci., New York, 1985, pp. 323-327. 
3. P. T. Bateman and P. Erdös, Geometrical extrema suggested by a lemma of Besicovitch, Amer. Math. Monthly 58 (1951), 306-314.

4. A. S. Besicovitch, $A$ general form of the covering principle and relative differentiation of additive functions (I), (II), Proc. Cambridge Philos. Soc. 41 (1945), 103-110; 42 (1946), $1-10$.

5. K. Bezdek and R. Connelly, Intersection points, Ann. Univ. Sci. Budapest Sect. Math. 31 (1988), 115-127.

6. J. Bliedtner and P. A. Loeb, A reduction technique for limit theorems in analysis and probability theory, Ark. Mat. 30 (1992), 25-43.

7. B. Bollobás, Graph theory, Springer-Verlag, New York, 1979.

8. H. Edelsbrunner, G. Rote, and E. Welzl, Testing the necklace condition for shortest tours and optimal factors in the plane, Theoret. Comput. Sci. 66 (1989), 157-180.

9. L. Fejes Tóth and A. Heppes, $A$ variant of the problem of the thirteen spheres, Canad. Math. Bull. 19 (1967), 1092-1100.

10. Z. Füredi, J. Lagarias, and F. Morgan, Singularities of minimal surfaces and networks and related extremal problems in Minkowski space, DIMACS Ser. Discrete Math. Theoret. Comput. Sci., vol. 6, Amer. Math. Soc., Providence, RI, 1991, pp. 95-109.

11. P. Gritzmann and J. Wills, Finite packing and covering, Studia Sci. Math. Hungar. 21 (1986), 149-162.

12. F. Harary, M. S. Jacobson, M. J. Lipman, and F. R. McMorris, On abstract sphere-ofinfluence graphs, J. Comput. Geom. Theory Appl. (submitted).

13. G. A. Kabatjanskiǐ and V. I. Levenštein, Bounds for packings on a sphere and in a space, Problemy Peredaci Informatsii 14 (1978), 3-25; English transl., Problems Inform. Transmission 14 (1978), 1-17.

14. D. T. Lee, Relative neighborhood graphs in the $L_{1}$-metric, Pattern Recognition 18 (1985), 327-332.

15. P. A. Loeb, On the Besicovitch Covering Theorem, Science University of Tokyo J. Math. 25 (1989), 51-55.

16. _ An optimization of the Besicovitch covering, Proc. Amer. Math. Soc. 118 (1993), 715-716.

17. V. D. Milman, Almost Euclidean quotient space of subspaces of finite dimensional normed spaces, Proc. Amer. Math. Soc. 94 (1985), 445-449.

18. A. P. Morse, Perfect blankets, Trans. Amer. Math. Soc. 61 (1947), 418-442.

19. G. Pisier, The volume of convex bodies and Banach space geometry, Cambridge Tracts in Math., vol. 94, Cambridge Univ. Press, Cambridge, 1989.

20. E. F. Reifenberg, A problem on circles, Math. Gaz. 32 (1948), 290-292.

21. C. E. Shannon, Probability of error for optimal codes in a Gaussian channel, Bell System Tech. J. 38 (1959), 611-656.

22. J. M. Sullivan, Sphere packings give bound for the Besicovitch covering theorem, J. Geom. Anal. (to appear).

23. H. P. F. Swinnerton-Dyer, Extremal lattices of convex bodies, Proc. Cambridge Philos. Soc. 49 (1953), 161-162.

24. G. T. Toussaint, Computational geometric problems in pattern recognition, Pattern Recognition Theory and Application (J. Kittler, ed.), NATO Advanced Study Institute, Oxford University, 1981, pp. 73-91.

25. I. M. Yaglom and V. G. Boltyanskiĭ, Convex figures, Holt, Rinehart, and Winston, New York, 1961.

26. L. Guibas, J. Pach, and M. Sharir, Sphere-of-influence graphs in higher dimensions, Intuitive Geometry (K. Böröczky and G. Fejes Tóth, eds.), Proc. Colloq. Math. Soc. J. Bolyai, North-Holland, Amsterdam (to appear).

27. S. G. Krantz and T. D. Parsons, Antisocial subcovers of self-centered coverings, Amer. Math. Monthly 93 (1986), 45-48. 
28. André E. Kézdy and Grzegorz Kubicki, $K_{12}$ is not a closed sphere-of-influence graph (to appear).

29. A. Malnić and B. Mohar, Two results on an antisocial families of balls, Proc. of the Fourth Czechoslovakian Sympos. on Combinatorics, Graphs and Complexity (Prachatice, 1990) (J. Nesetril and M. Friedland, eds.), Elsevier, New York; Ann. Discrete Math. 51 (1992), 205-207.

30. T. S. Michael and T. Quint, Sphere of influence graphs: edge density and clique size, manuscript, 1994.

Department of Mathematics, University of Illinois, 1409 West Green St., Urbana, ILLINOIS 61801-2917

E-mail address: zoltan@math.uiuc.edu

E-mail address: loeb@math.uiuc.edu 